\title{
Self-Powered Kinetic Energy Harvesters for Seek-Induced Vibrations in Hard Disk Drives*
}

\author{
Jen-Yuan (James) CHANG** and Mike GUTIERREZ*** \\ ${ }^{* *}$ School of Engineering and Advanced Technology \\ Massey University \\ Albany, Auckland, 0745, New Zealand \\ E-mail: J.Y.Chang@massey.ac.nz \\ ***Hitachi Global Storage Technologies, San Jose, CA, USA
}

\begin{abstract}
Energy harvesters with battery charging circuitry, which collect wasted kinetic energy from a magnetic disk drive's rotary actuator seek operations and flexible cable vibrations, are proposed, prototyped and presented in this paper. Depending on a disk drive's form factor and seek format, it is suggested by the present study that the harvested energy can be optimized by tuning the harvester's natural frequencies to major frequency content in the rotary actuator's excitation. It is demonstrated in this study that with prototype energy harvester systems, one can easily light up a regular LED. The work presented in this paper has implications in energy saving and recycling wasted mechanical energy for other low-power electronic applications in magnetic disk drive storage devices.
\end{abstract}

Key words: Energy Harvester, HDD, Kinetic Energy, Self-Power Device, Seek-Induced Vibrations, Rotary Actuator.

\section{Motivation}

In twenty-first century, information and energy can be viewed as the two most important enablers in each individual's daily life. We go online for emails, news, driving directions, reserving trips, paying bills, etc. Each mentioned activity is highly related to information storage as well as processing the obtained information. With increasing human population, the demand of energy to transform materials to maintain human's needs increases drastically disregarding limited resource available world-wide. This demand on the other hand suggests immediate need to engineer ways and methods to sustain usage of energy.

In information age, a magnetic hard disk drive (HDD) is the common and widely used engineered mechatronic device ${ }^{(1)}$ for information storage and processing of existing information for other usage. As illustrated in Fig. 1 (a), various form factors including 1-inch, 2.5-inch, and 3.5-inch can be used for a HDD to suite for different applications. Common applications for a HDD include personal information storage and data computation in laptop or desktop personal computers, information storage units in a data storage center or an enterprise server, as well as endless list of consumer electronic products such as camcorders, global position systems (GPS), cell phones, iPods, personal digital assistants (PDA), and handheld gaming devices.

When a HDD is used in the above mentioned applications, to access data, its rotary actuator as exampled in Fig. 1 (b) and Fig. 1 (c), which carries magnetic read and write elements, needs to perform seek operations toward targeted data tracks on magnetic disks. The seek operations are achieved by alternating amplitude and direction of supply current in HDD's voice coil motor (VCM). As a result of the alternating current through the flex 
cable, the electrical energy is transformed electro-magnetically into mechanical kinetic energy to swing the rotary actuator toward targeted tracks.

Although this kinetic energy successfully moves the read/write elements on a rotary actuator to desired locations on disks, from literatures ${ }^{(2)-(3)}$, it also results in undesirable actuator and flex cable dynamics in form of excessive position error signals which hinder a HDD's data access performance. The generated actuator and flex cable dynamics are commonly in form of wasted mechanical kinetic energy which eventually is then transformed into heat later dissipated into surrounding environment. As a regular HDD is regularly performing seeking operations, given the above mentioned facts and to address energy saving for "greener" data storage products, the focus of our research presented in this paper is to provide a proof-of-concept design and apparatus that can convert the wasted kinetic energy due to actuator seek operations into re-useful electrical form.

(a)

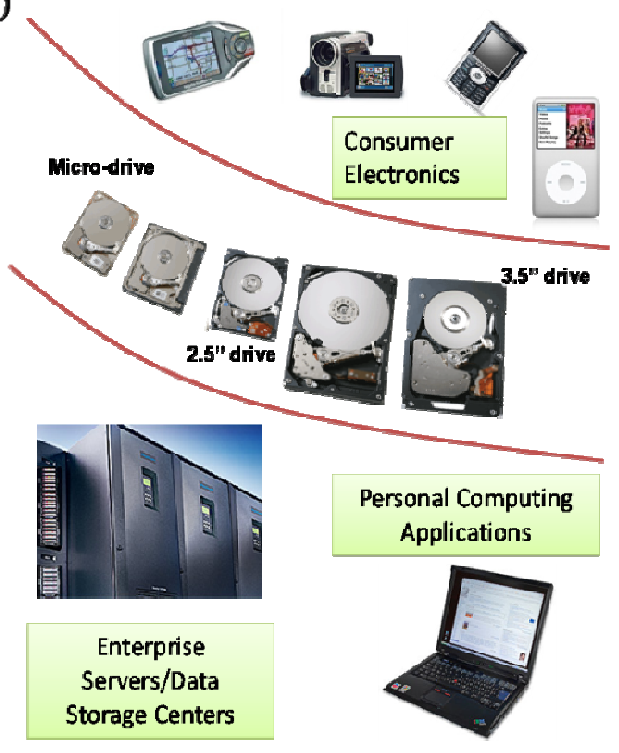

(b)

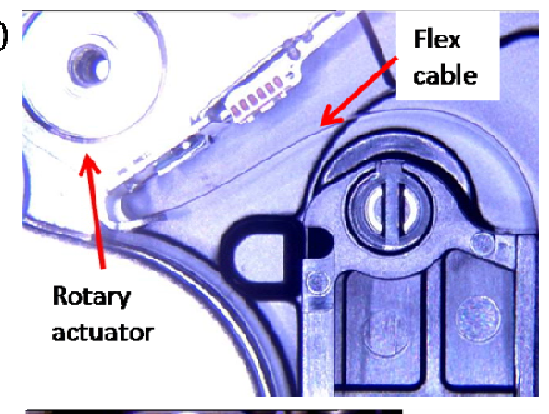

(c)

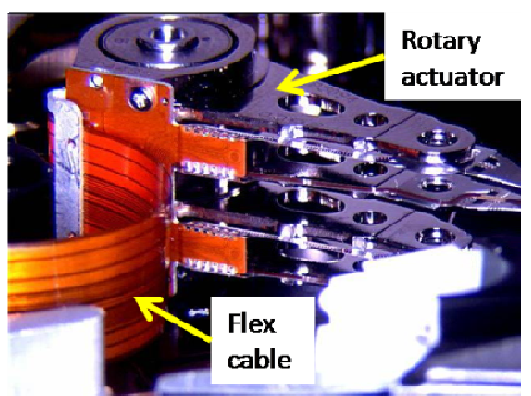

Fig. 1 (a) Applications of commercial hard disk drives. (b) Top view and (c) side view of a rotary actuator-flex cable system in a HDD.

\section{Review of Common Energy Harvesting Methods}

Energy harvesting which is commonly known as energy scavenging is a process to capture and store energy that is generated by external sources. It was initiated by Defense Advanced Research Projects Agency (DARPA) of USA through industry-academic collaborated projects such as Heel-Strike Generator for Soldiers and Energy Harvesting Eel for Undersea Power Generation from 1995 to early 2000s. It was reported that by proper designs, about $35 \%-65 \%$ of elastic or wasted mechanical energy can be transformed into electrical power to power cooling devices for soldiers ${ }^{(4)}$. Although percentage of actual harvested energy can vary from applications to applications, the concept of energy harvesting for power has drawn significant attentions from industry and public sectors ${ }^{(5)}$.

In addition to widely known solar/light, geothermal, and hydro energy harvesting techniques, commonly seen miniature energy harvesters can be cataloged functionally into harvesting of radiation energy, biomechanical energy, electrostatic energy, electromagnetic energy, and piezoelectric energy. Operation of a radiation energy harvester is similar to circuitry of a radio ${ }^{(6)}$. The harvester identifies specific broadcasted radio frequencies from ambient environment and then transforms the energy to power remote devices. Operation method of a biomechanical energy harvester is exampled by Donelan et al.'s apparatus and 
system ${ }^{(7)}$ that transforms energy generated by muscle during human walking into electricity to power portal medical devices. Operation principle of an electrostatic energy harvester is quite similar to storing electrons in a capacitor except that the capacitance is changed in vibration-dependent varactors, commonly known as variable capacitor, due to coupled external mechanical motions ${ }^{(8)}$. Electromagnetic energy harvesters ${ }^{(9)-(10)}$ simply adopt Faraday's law while piezoelectric energy harvesters use piezoelectric effect ${ }^{(11)-(16)}$ to transform mechanical motions into electricity. To obtain optimal electric energy output, both electromagnetic and piezoelectric energy harvesters need to operate at or close to their mechanical resonant modes.

\section{Kinetic Energy Harvesting System for Seek-Induced Vibrations}

To avoid magnetic interference and electrostatic discharge inside a HDD, in this research, piezoelectric-type of energy harvester is chosen for prototype development. Two energy harvesters, namely actuator kinetic energy harvester (AKEH) and flexible-cable kinetic energy harvester (FKEH), which are developed to collect wasted kinetic energy during HDD's seek operations from rotary actuator and flexible-cable, respectively, will be discussed in details in this section.

To demonstrate feasibility of harvesting wasted kinetic energy in a HDD, the kinetic energy harvesting system as depicted in the schematics in Fig. 2 below excludes energy storage unit commonly seen in energy harvesters reported in the literatures. Instead of storing electric energy into batteries, a light-emitting diode (LED) is first used as an immediate indication of harvested kinetic energy into electric form. The energy harvesting system consists of two converters, namely, a mechanical to electrical converter unit and an alternating current (AC) to direct current (DC) converter unit.

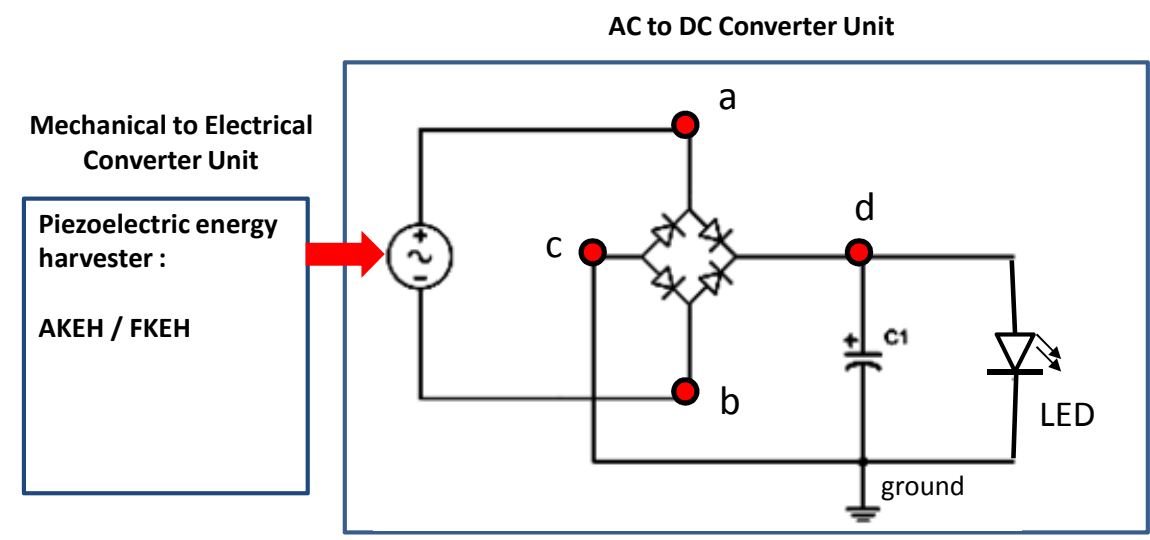

Fig. 2 Schematics of a HDD kinetic energy harvesting system.

Designs of the mechanical to electrical converter unit incorporating an AKEH or a FKEH will be elaborated later. Due to the mechanical nature of seek operations, electrical output from both AKEH and FKEH is expected and in fact observed to be in form of an alternating current having non-sinusoidal oscillating patterns. To avoid cancellation of negative and positive electrons generated by either AKEH or FKEH and to minimize forward voltage drop, a full wave rectifier is constructed in the AC to DC converter unit using four identical Schottky diodes. The $\mathrm{C} 1$ capacitor is chosen so as to accumulate enough electric potential to overcome forward voltage bias of 1.4-2.0 Volts commonly inherited in a green LED. Realization of such AC to DC converter unit is shown in photographs in Fig. 3 where a green LED as shown in Fig. 3 (b) is lighted up using harvested kinetic energy when a HDD rotary actuator is conducting seek operations. To investigate how much energy can be harvested in a seek-performing HDD, the green LED is 
then replaced by a $2.4 \mathrm{~V}, 90 \mathrm{mAh}$, nickel-metal hydride $(\mathrm{NiMH})$ rechargeable battery as shown in Fig. 3 (b).

(a)

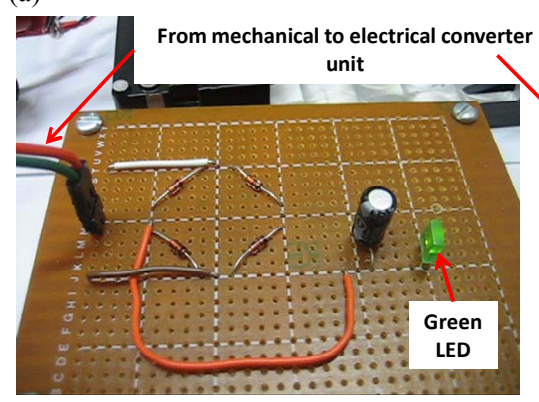

(b)

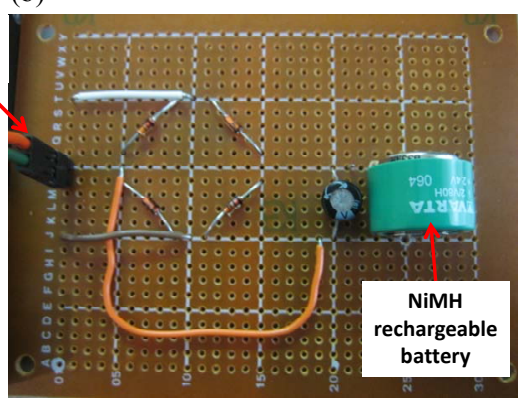

Fig. 3 Photograph of AC to DC converter unit together with (a) a green LED and (b) a NiMH rechargeable battery.

\section{Actuator Kinetic Energy Harvester (AKEH)}

Design of a prototype AKEH is discussed in this section. Organization of this section starts from overall system design considering kinematics of a rotary actuator-flex cable system in a 3.5 " commercial hard disk drive (Hitachi Ultrastar 15K300) followed by design and modeling of AKEH and then closed by experimental measurements.

(a)

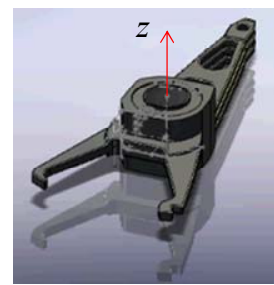

(d)

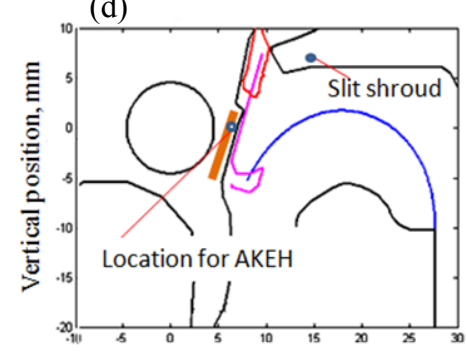

Horizontal position, $\mathrm{mm}$ (b)

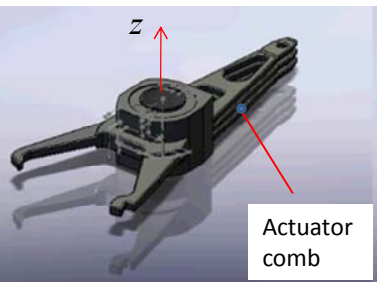

(e) (c)

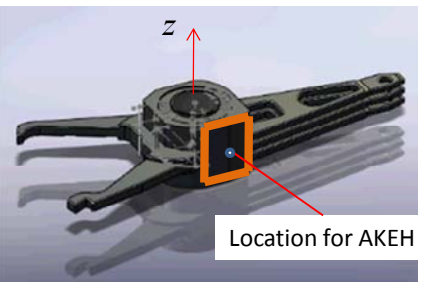

(f)

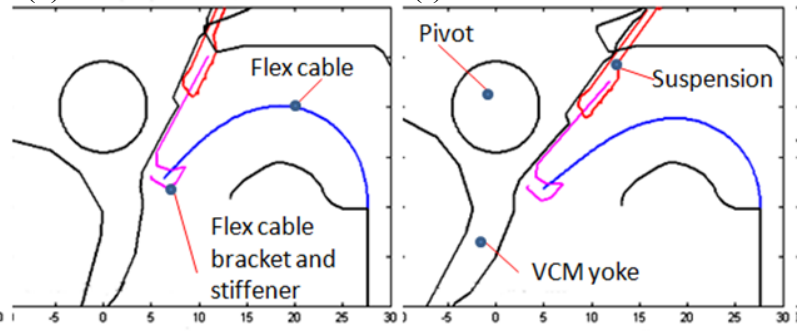

Horizontal position, $\mathrm{mm}$

Fig. 4 Simulated rotary actuator comb kinematics at (a) ID, (b) MD, and (c) OD orientations. Schematics of the rotary actuator-flex cable system profiles at (d) ID, (e) MD, and (f) OD orientations, respectively.

\subsection{Kinematics of Rotary Actuator-Flex Cable System}

For illustration purpose, kinematics of a rotary actuator comb (excluding suspensions for simplicity) is simulated using COSMOS Motions in SolidWorks as shown in Fig. 4 (a)-(c) using realistic geometric values from the HDD when its rotary actuator is rotated with respect to $z$-axis and oriented toward disk's inner diameter (ID, $67^{\circ}$ from horizontal axis), mid-diameter (MD, $54^{\circ}$ from horizontal axis), and outer diameter (OD, $41^{\circ}$ from horizontal axis), respectively. Using elastic model ${ }^{(3)}$ for flexible cable's large deformation, the corresponding cable profiles at ID, MD, and OD orientations are illustrated in Fig. 4 (d), (e), (f), respectively. For the model commercial 3.5-inch HDD, physical distance between ID and OD tracks is about $25 \mathrm{~mm}$ and distance between read/write 
element and the pivot center is $30 \mathrm{~mm}$. That is, the full-stroke rotation angle for the actuator to move read/write elements from OD track to ID track is $\theta_{F S}=25 / 30=0.83 \mathrm{rad}$. Suppose $t_{F S}$ is the move time in seconds required for the rotary actuator to perform full-stroke seek and $J_{a c t}=1.3 \times 10^{-6} \mathrm{~kg}-\mathrm{m}^{2}$ is rotary inertia of the entire rotary actuator system with respect to $z$-axis, the kinetic mechanical energy for the rotary actuator under full-stroke seek can be expressed in the following equation.

$$
T_{F S}=\frac{1}{2} J_{a c t}\left(\frac{\theta_{F S}}{t_{F S}}\right)^{2} \approx \frac{4.5 \times 10^{-7}}{t_{F S}{ }^{2}}
$$

Obviously from equation (1), with faster move time, higher kinetic mechanical energy can be produced. The corresponding mechanical power, which is the work done per second as defined as $P_{a c t}=\mathrm{T}_{\mathrm{FS}} / t_{F S}$, is the result of engagement of VCM current in VCM's predefined magnetic fields. With coil resistance for the model HDD VCM being $R=5.76 \mathrm{Ohms}$, VCM current denoted as $v(t)$, and taking $\mathrm{VCM}$ current being an AC into consideration, the averaged VCM power to allow the actuator to perform a full stroke seek becomes

$$
P_{V C M}=\frac{1}{t_{F S}} \int_{0}^{t_{F S}} \frac{v(t)^{2}}{R} d t .
$$

When the rotary actuator undergoes full-stroke seek from OD to ID, as illustrated in Fig. 4 (d)-(f), the flex cable, which connects HDD's printed circuit board and the rotary actuator, significantly changes its profile. This drastic profile change contains rich kinetic mechanical energy which will be addressed later in separate section.

\subsection{A Design of AKEH for Manufacturing Compatibility}

The geometry and mechanical design of a rotary actuator in a HDD has been significantly engineered in the past two decades by researchers and engineers to allow the actuator to move to targeted tracks on magnetic disks in a timely manner but also with high precision accuracy and high servo stability for data tracking. To include an energy harvester to existing high precision data storage device like a HDD, careful examinations for available options were first conducted with view toward defining package size and location on the rotary actuator.

(a)

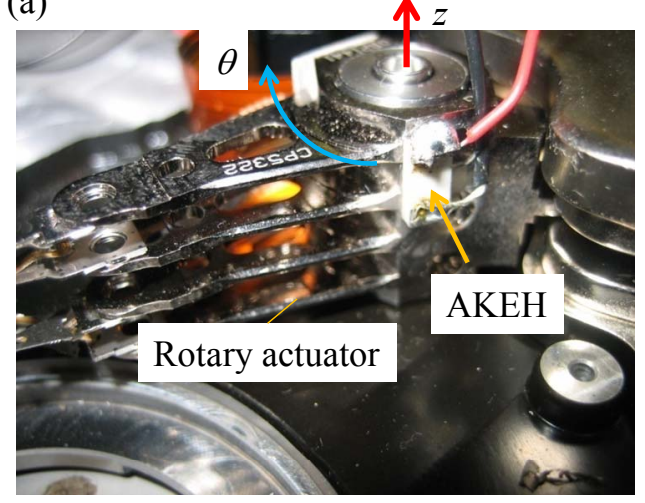

(b)

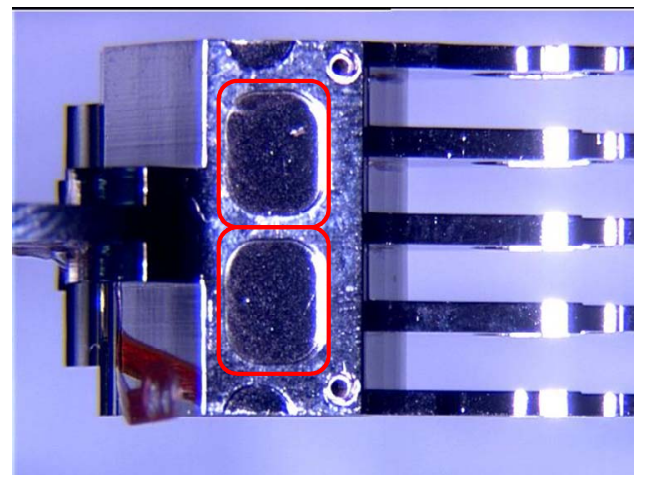

Fig. 5 (a) Example of placing an AKEH on a rotary actuator and (b) available pocketed space indicated by red rectangles on flex cable stiffener side for AKEH to be embedded in the actuator comb structure.

As one can observe from actuator pictures shown in Fig. 1(c), Fig. 4 (a)-(c), and Fig. 5(a), the actuator comb, which are connected to suspensions, and VCM yoke, which carries voice coils, are structures that need to move between mechanical parts such as spinning disks and VCM magnets for comb and yoke, respectively. By installing an extra 
component on actuator comb or yoke can first introduce risk for mechanical interference during seek operations. Secondly it can cause mechanical imbalance which certainly jeopardizes actuator servo tracking stability. Therefore, the only place to install the AKEH is in the comb structure near pivot as indicated by orange rectangle in Fig. 4(c) and red rectangles in Fig. 5(b). For an illustrative example, a prototype AKEH having package dimensions of $4 \mathrm{~mm}$ in height, $2 \mathrm{~mm}$ in width, and $1 \mathrm{~mm}$ in depth is installed on comb surface as shown in Fig. 5 (a). However, in our final testing, the AKEH is embedded in one of the two red rectangles as shown in Fig. 5(b). Installing the AKEN in one of the red rectangle pocket has implication on manufacturing compatibility for flex cable and actuator comb. This is because the flex cable's bracket and stiffener as indicated in Fig. 4(e) are fact installed on this surface. If one can carefully design flex cable circuitry on the stiffener, the AKEH can be easily connected to flex cable without extra wires. The AC to DC converter unit as shown in Fig. 2 can be either put on flex cable's stiffener or on HDD's printed circuit board.

\subsection{A PZT-based AKEH as a Vibration Absorber}

A bimorph zirconate titanate $\mathrm{Pb}(\mathrm{Zr}$ Ti $) \mathrm{O}(\mathrm{PZT})$ cantilever beam structure carrying a lumped inertia $J_{p}$ at its end with dimensions of $W=0.2 \mathrm{~mm}, h=60 \mathrm{um}$, and $L=2 \mathrm{~mm}$ as sketched in Fig. 6(a) is designed as the AKEH to capture actuator seek kinetic energy. The corresponding spring-inertia equivalence is shown in Fig. 6(b) where $k_{a c t}$ and $k_{p}$ are equivalent stiffness for the rotary actuator and the PZT beam under VCM's in-plane seek excitation $T(t)$, respectively.

(a)

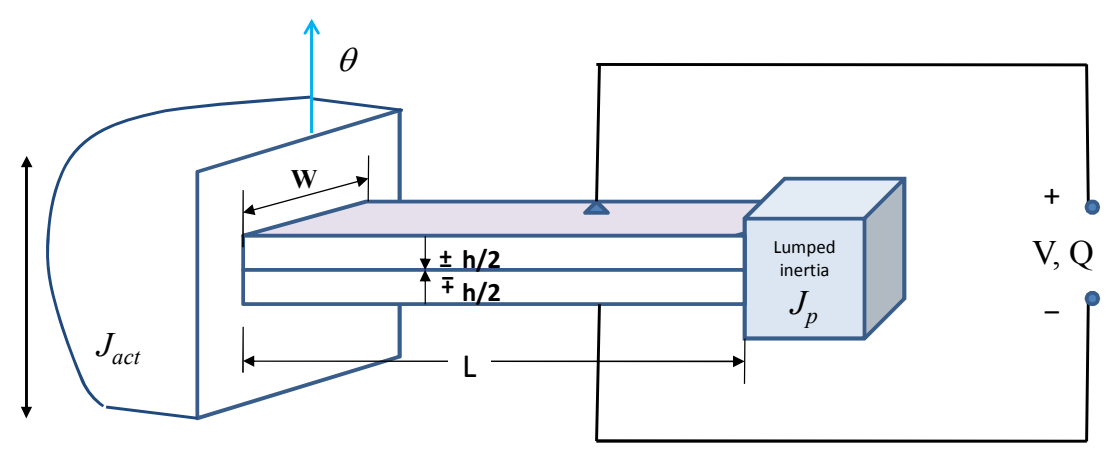

(b)

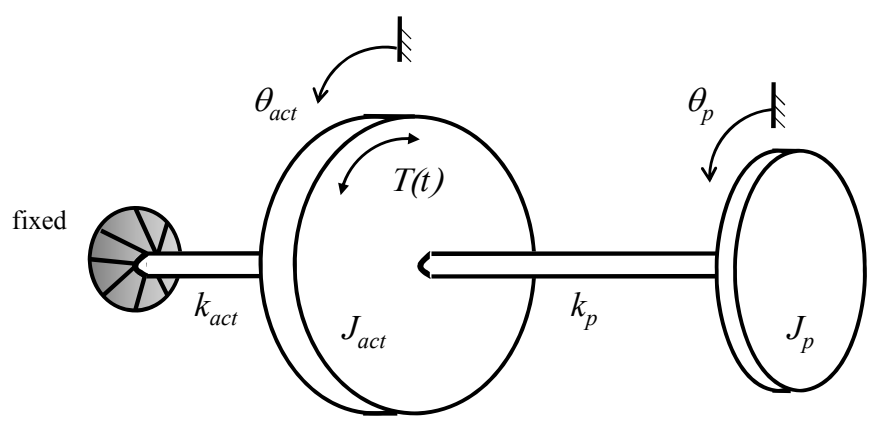

Fig. 6 (a) Schematics of beam-like PZT AKEH and (b) the corresponding two degree-of-freedom spring-inertia system under torsional load $T(t)$.

Following Uchino's classical electromechanical coupling model ${ }^{(14)}$, the output voltage $V$ and charge $Q$ can be expressed as:

$$
\begin{aligned}
& V=\frac{3 T_{d} L g_{31}}{2 W h} \\
& Q=\frac{3 T_{d} L^{2} d_{31}}{2 W h}
\end{aligned}
$$


where $T_{d}=J_{p} \ddot{\theta_{p}}$ is the dynamic load on the lumped inertia due to actuator excitation and $g_{31}=9.0 \times 10^{-3} \mathrm{Vm} / \mathrm{N}$ and $d_{31} \approx 280 \times 10^{-12} \mathrm{~m} / \mathrm{v}$ are piezoelectric charge and piezoelectric voltage constants resulted in $\theta$ direction, respectively. From equations (3) and (4), in order to optimize harvested energy, one needs to ensure optimal value of $T_{d}$. That is in other words, the AKEH lumped inertia cantilever beam needs to be tuned at or close to its resonant frequencies during seek operations.

The design concept of the present AKEH follows classical vibration absorber as concept described by Rao ${ }^{(17)}$. Based on the equivalent spring-inertia system as shown in Fig. 6(b) using Newtonian approach, equations of motion for the two degree-of-freedom system are:

$$
\begin{aligned}
& J_{a c t} \ddot{\theta_{a c t}}=k_{a c t} \theta_{a c t}-k_{p}\left(\theta_{a c t}-\theta_{p}\right)+T(t) \\
& J_{p} \ddot{\theta_{p}}=k_{p}\left(\theta_{a c t}-\theta_{p}\right)
\end{aligned}
$$

Applying Laplace transform on equation (5) and (6), response transfer function in frequency $s$ domain for actuator becomes

$$
\frac{\theta_{a c t}(s)}{T(s)}=\frac{J_{p} s^{2}+k_{p}}{\left(J_{a c t} s^{2}+k_{a c t}+k_{p}\right)\left(J_{p} s^{2}+k_{p}\right)-k_{p}^{2}} .
$$

While for the lumped inertia of AKEH, its response displacement to excitation ratio is

$$
\frac{\theta_{p}(s)}{T(s)}=\frac{k_{p}}{\left(J_{a c t} s^{2}+k_{a c t}+k_{p}\right)\left(J_{p} s^{2}+k_{p}\right)-k_{p}^{2}} .
$$

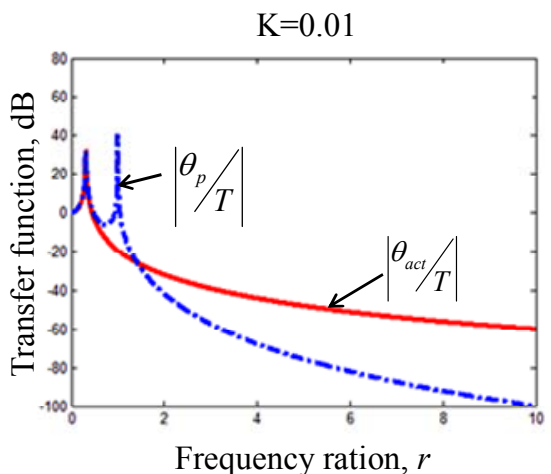

$\mathrm{K}=3.5$

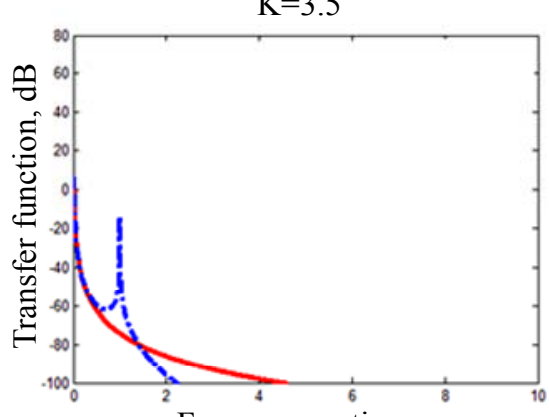

Frequency ration, $r$

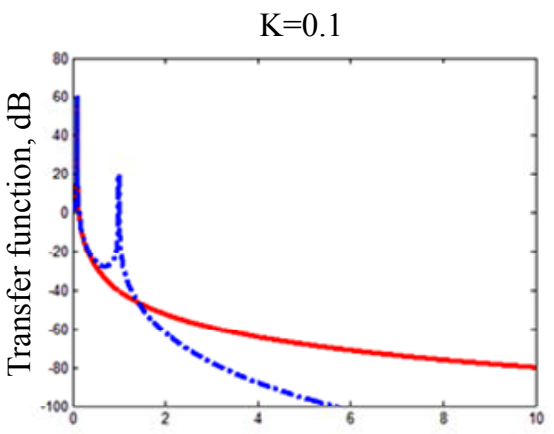

Frequency ration, $r$

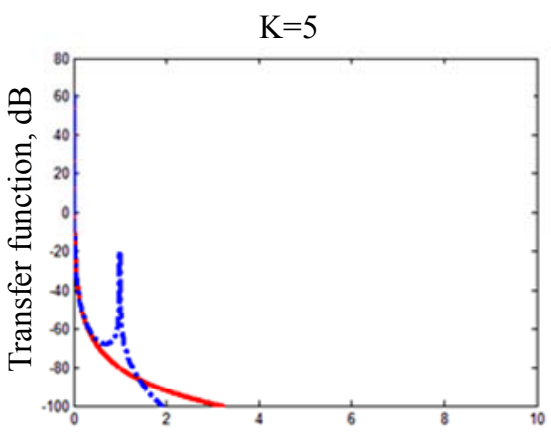

Frequency ration, $r$

Fig. 7 Comparison of response transfer functions as a function of frequency ration $r$ at $\mathrm{K}=0.01,0.1$, 2.5 , and 5 for the actuator-AKEH system.

Defining stiffness ration $\mathrm{K}=\mathrm{k}_{\mathrm{p}} / \mathrm{k}_{\text {act }}$ and frequency ratio $r=\omega / \omega_{p}$, where $\omega$ and $\omega_{p}$ are VCM excitation frequency and first bending mode natural frequency of the AKEH system, 
transfer functions for the rotary actuator and the AKEH are plotted in Fig. 7 at $\mathrm{K}=0.01,0.1$, 2.5, and 5. For a typical 3.5-inch HDD, the fundamental in-plane flexure vibration mode for rotary actuator is about $10 \mathrm{kHz}$. For the present application, the $1^{\text {st }}$ bending mode of the AKEH is intentionally tuned at $35 \mathrm{kHz}$ to be decoupled from major actuator dynamics. As exampled in Fig. 7 , when $\mathrm{K}=0.01$, the AKEH is not absorbing the majority of the actuator kinetic energy while when $\mathrm{K}=3.5$ and $\mathrm{K}=5$, the $\mathrm{AKEH}$ can pretty much capture majority of actuator kinetic energy if the VCM excitation is tuned to the AKEH's resonant frequency $\omega_{p}$.

\subsection{Measurements}

A PZT material is commonly modeled as a capacitor in electrical circuit ${ }^{(11)-(16)}$ primary due to its piezoelectric effect. Voltage measurements using a HP oscilloscope are compared in Fig. 8 followed by connecting the prototype AKEH to the AC to DC converter unit as indicated in Fig. 2. Without VCM excitation, result shown in Fig. 8(a) is a typical capacitor charging and de-charging signature. Voltage output from the AKEH is measured across point $\mathrm{a}$ and point $\mathrm{b}$ as shown in Fig. 2. This output, which is before the fall wave rectifier is plotted in Fig. 8(b) when the VCM is being excited at $4 \mathrm{kHz}$. If one measures voltage across point $\mathrm{c}$ and point $\mathrm{d}$ without the capacitor $\mathrm{C} 1$ in Fig. 2, the negative charges are rectified by the rectifier which is plotted in Fig. 8(c). Measuring with a $\mathrm{C} 1=6.8 \mathrm{uF}$ capacitor in Fig. 2, the final output voltage as shown in Fig. 8(d) is found to be DC at $500 \mathrm{mV}$ level.

(a)

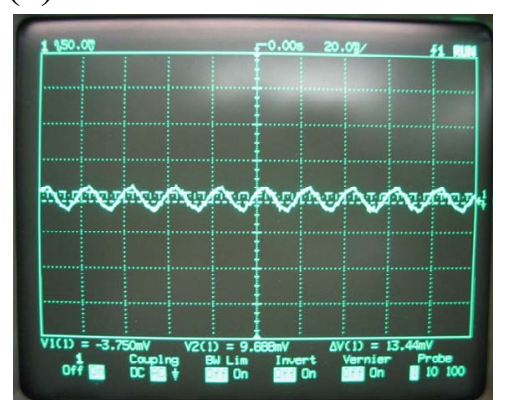

(c)

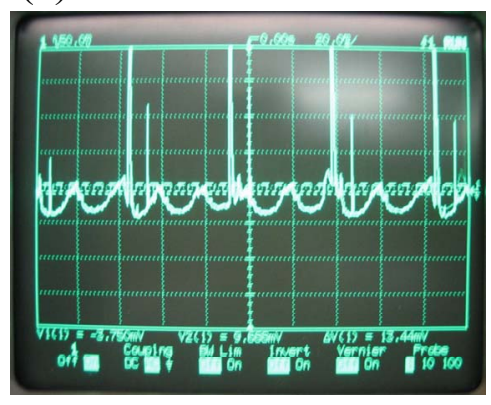

(b)

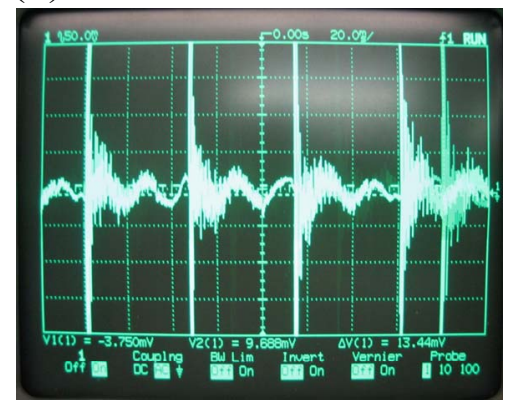

(d)

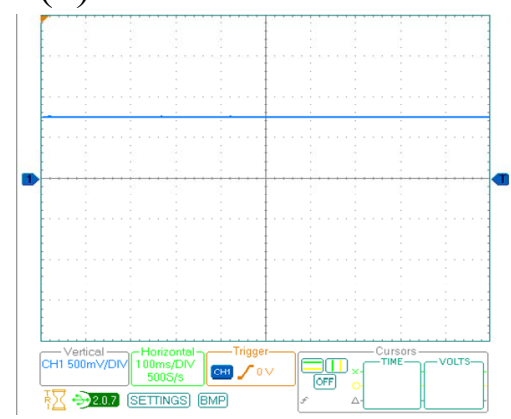

Fig. 8 Comparison of charging voltage from energy harvester: (a) w/o VCM excitation; (b) with VCM excitation, $V_{a b}$ and (c) with VCM excitation, $V_{c d}$.

Obviously, one can choose different type of capacitors for different applications. To power a small electronic such as the green LED shown in Fig. 3(a), a SMD $4.7 \mathrm{uF} / 5 \mathrm{~V}$ capacitor was used. It was found that the LED can be light up easily by the AKEH when VCM excitation is tuned to 10 to $40 \mathrm{kHz}$. As mentioned in previous section in this paper, optimal voltage output from the AKEH can be achieved if the AKEH can act as vibration 
absorber in the rotary actuator-AKEH system. To verify the theoretical prediction with the prototype AKEH's first bending mode frequency being aroun $35 \mathrm{kHz}$, an experiment was conducted from which harvested voltage $V_{c d}$ was measured at increasing VCM excitation frequency as shown in Fig. 9 below. From the figure, it is apparent that maximum voltage can be created by the AKEH when VCM is driving the rotary actuator around $35-38 \mathrm{kHz}$. In other words, one can simply design the AKEH's cantilever beam resonant modes to major VCM excitation frequencies for optimal kinetic energy harvesting. Driving at 35 $\mathrm{kHz}$ with the prototype AKEH and the model HDD, it was found the empty $2.4 \mathrm{~V}, 80 \mathrm{mAh}$, NiMH rechargeable battery as shown in Fig. 3(b) can be fully charged in about 1.5 hours.

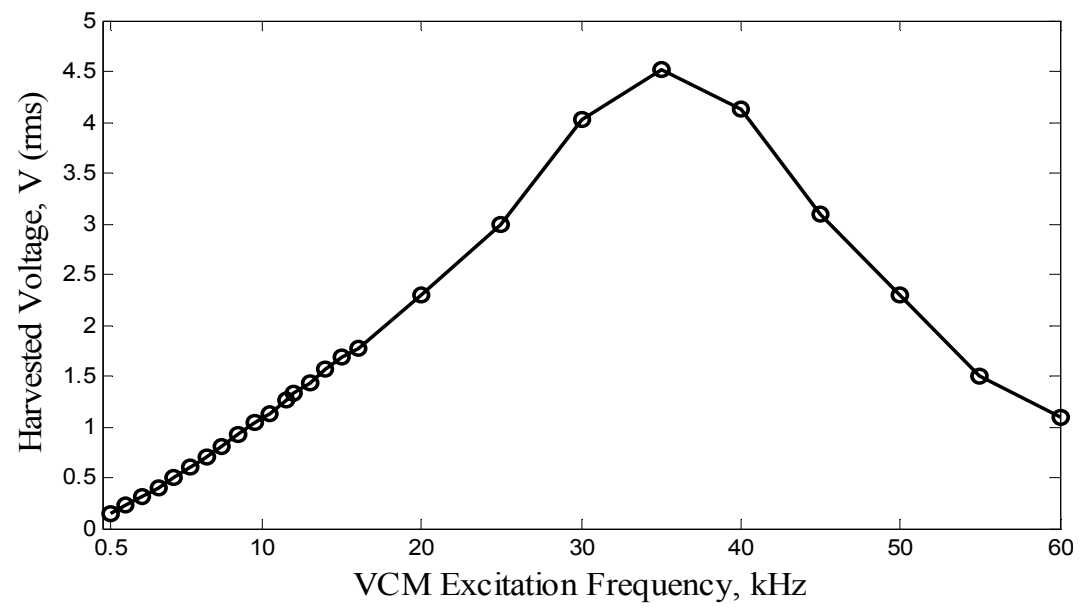

Fig. 9 AKEH voltage output as a function of VCM excitation frequency.

Using equation (1) and (2) for energy calculation, with VCM excitation tuned to the AKEH's resonant mode at $35 \mathrm{kHz}$, about $20 \%$ of electrical energy is lost when it is transformed into mechanical kinetic energy. However, it was found that $40 \%$ of the mechanical kinetic energy would be able to be harvested by the AKEH back into electrical energy form.

\section{Flexible-Cable Kinetic Energy Harvester (FKEH)}

Accodring to the principle of conservation of energy, all energy forms added up should be conserved. Therefore, the next objective of our research was to find out the second biggest kinetic enegry generator. As illustrated in Fig. 4(d)-(f), during actuator seek motions, the flex cable's profile is signifncantly changed. Although flex cable inertia is not as big as that of the rotary actuator, its dynamic displacement is much bigger than actuator's which can also result in significant dynamic energy. To prove the concept, a piezoelectric Polyvinylidene Fluoride (PVDF) as shown in pictures in Fig. 10 below is used as the flexible-cable kinetic energy harvester (FKEH) to collect kinetic energy from the flex cable. The film is attached to the flex cable using doubled sided taype.
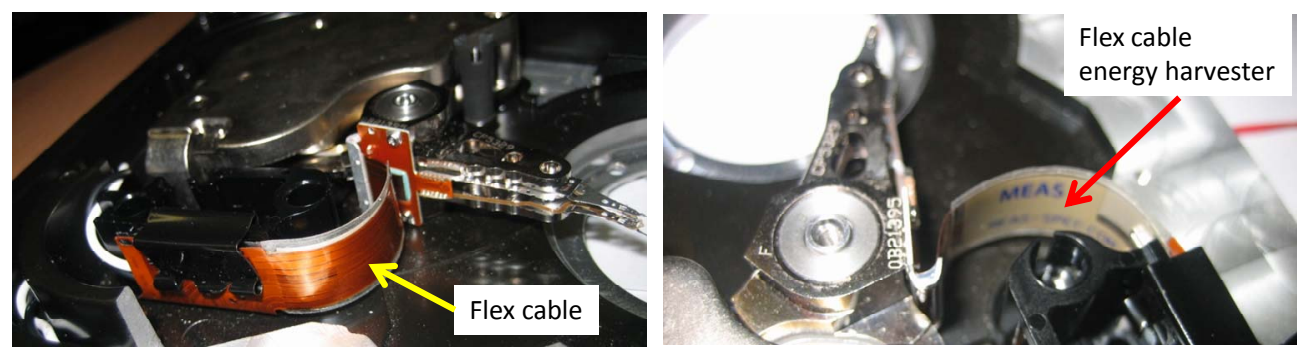

Fig. 10 Placement of FCEH on flexible-cable.

Along with the AKEH at $35 \mathrm{kHz}$ VCM excitation, it was found that energy harveted by the FKEH is about $10 \%$ of the actuator mechanical kinetic energy. Since suspensions as 
well as actuator comb and yoke structures can undergo dynamic deformation, further kinetic energy can be harvested by attaching film-like energy harvesters on the mentioned actuator components.

\section{Conclusions}

Two energy harvester prototypes AKEH (actuator kinetic energy harvester) and FKEH (flexible-cable kinetic energy harvester) for actuator and flexible cable kinetic energy harvesting, respectively were designed, developed and tested in a commercial 3.5-inch mechanical hard disk drive. Feasibility of using the energy harvesting systems to convert seek-induced mechanical kinetic energy into electrical energy is demonstrated. A vibration absorber design approach is used in the AKEH design for optimal kinetic energy harvesting. With VCM excitation being tuned to AKEH's $1^{\text {st }}$ bending mode, about $40 \%$ of the actuator kinetic energy is harvested by the AKEH and $10 \%$ by the FKEH. Although further optimization considering disk drive's form factor, manufacturing compatibility, and servo seek format is implied, the present study suggests an alternative way of reducing power consumption in hard disk drives by recycling wasted mechanical kinetic energy into electrical form. The work presented in this paper suggests an alternative renewable energy from wasted seek-introduced kinetic energy in hard disk drives. The harvested energy not only can be used to power small electronic devices but also can be accumulated to perform other application such as cooling of overheated severs in data storage centers.

\section{Acknowledgement}

The first author gratefully acknowledges the financial support and donation of hard disk drives by Hitachi Global Storage Technologies, San Jose, CA, USA. Valuable technical discussions with Mr. Robert Reinhart and Mr. Yogeshi Doshi for the work described wherein have been highly appreciated. This work is also partially supported by start-up fund under account number PR97384 by Massey University, New Zealand.

\section{References}

(1) Chang, J.Y., Hard Disk Drive Mechatronics: Servo, Mechanical, and Manufacturing, Keynote Speech of 15th International Conference on Mechatronics and Machine Vision in Practice, December 3 (2008), Auckland, New Zealand.

(2) Chang, J.Y., Hard Disk Drive Seek-Arrival Vibration Reduction with Parametric Damped Flexible Printed Circuits, Journal of Microsystem Technologies, Vol. 13 (2007), pp.1103-1106.

(3) Chang, J.Y., Minimization of Flexible Cable Settling PES in High TPI Hard Disk Drive via Mechatronic Integration, IEEE Transactions on Magnetics, Vol. 45 (2009), No. 11-1, pp. 4956-4961.

(4) Nowak, R.J., DARPA's Advanced Energy Technologies, Proceedings of DARPA 21th Systems and Technology Symposium, September 6-8 (2000), Dallas, TX, USA.

(5) Harrop, P. and Das, R., Energy Harvesting and Storage for Electronic Devices 2009-2019, (2009), IDTechEX.

(6) Cote, A. and Abadi, F., RF Release Mechanism for Hard Tag, July 21 (2009), United State Patent 4,564,360.

(7) Donelan, J.M., Li, Q., Naing, V., Hoffer, J.A., Weber, D.J., and Kuo, A.D., Biomechanical Energy Harvesting: Generating Electricity During Walking with Minimal User Effort, Science, Vol. 319 (2008), No. 5864, pp.807-810.

(8) Chen, K-S., Ou, K-S., Development and Verification of 2D Dynamic Electromechanical Coupling Solver for Micro-Electrostatic-Actuator Applications, Sensors and Actuators A: Physical, Vol. 136 (2007), Issue 1, pp.403-411.

(9) Wang, P., Tanaka, K., Sugiyama, S., Dai, X., Zhao, X., and Liu, J., A Micro Electromagnetic Low Level Vibration Energy Harvester Based on MEMS Technology, Journal of Microsystem Technology, Vol. 15 (2009), pp.941-951.

(10) Kulkarni, S., Koukharenko, E., Torah, R., Tudor, J., Beeby, S., O’Donnell, T., and 
Roy, S., Design, Fabrication and Test of Integrated Micro-Scale Vibration-Based Electromagnetic Generator, Sensors and Actuators A: Physical, Vol.145 (2007), pp.336-342.

(11) Williams C.B., Yates R.B., Analysis of a Micro-Electric Generator for Microsystems, Sensors and Actuators A: Physical, Vol. 52 (1996), pp.8-11.

(12) Umeda, M., Nakamura, K., and Ueha, S., Analysis of Transformed Mechanical Impact Energy to Electrical Energy using a Piezoelectric Vibrator, Japanese Journal of Applied Physics, Vol. 35 (1996), Part 1, No. 5B, pp.3267-3273.

(13) Umeda, M., Nakamura, K., and Ueha, S., Energy Storage Characteristics of a Piezo-Generator using Impact Induced Vibration, Japanese Journal of Applied Physics, Vol. 35 (1997), Part 1, No. 5B, pp.3146-3151.

(14) Uchino, K and Giniewicz, J.R., Micromechatronics (2003), Marcel Dekker, Inc., New York, NY, USA, ISBN: 0-824-4109-9.

(15) Sodano, H.A, Park, G, and Inman, D.J, A Review of Power Harvesting From Vibration using Piezoelectric Materials, Vol.C (2004), The Shock and Vibration Digest.

(16) Stephen, N.G., On Energy Harvesting from Ambient Vibration, Journal of Sound and Vibration, Vol.293 (2006), pp.409-425.

(17) Rao, S.S., Vibration of Continuous Systems, Wiley (2007), New York, ISBN 0417771716 